\title{
Left Atrial Infarction with Saddle Embolism
}

\author{
Hisayoshi Fujiwara, M.D., Hiroshi Saimyoj, M.D., \\ Chuichi Kawai, M.D., Fumitada Hazama, M.D., * and \\ Hideyuki Haebara, M.D.*
}

\section{SUMmary}

Left atrial and left ventricular infarction with various atrial arrhythmias, multiple systemic arterial thromboembolism, and a large mural thrombus over the left atrial infarction is reported.

In ischemic heart disease, systemic arterial thromboembolism may develop due to atrial infarction which is often overlooked in antemortem as well as in postmortem examinations.

When a clinical diagnosis of atrial infarction is made, the possibility of a Stokes-Adams syndrome, thromboembolism, and rupture of the atrium should also be investigated.

\section{Additional Indexing Words :}

Ischemic heart disease Supraventricular arrhythmias Systemic arterial thromboembolism Left atrial thrombus

\begin{abstract}
A TRIAL infarction has generally been considered as a rare lesion of little A clinical importance. ${ }^{\prime \prime}$ Saddle embolism in ischemic heart disease is usually attributed to left ventricular mural thrombi.

The purpose of this report is to present a case of left atrial infarction with saddle embolism due to a left atrial mural thrombus and to stress the clinical significance of atrial infarction.
\end{abstract}

\section{Case Report}

A 57-year-old Japanese female was transferred to our hospital on March 11, 1974 following a sudden onset of numbness, pain, and coldness in the bilateral lower extremities.

For the preceding 5 years the patient had had dyspnea, palpitation, and oppressive feeling in the chest on exertion. From 1973 on, she had experienced left back pain lasting from several minutes to half an hour after she had ingested some cold fluid. ECG occasionally showed multifocal supraventricular tachycardia

From the Third Division, Department of Internal Medicine, Faculty of Medicine, Kyoto University, Kyoto.

* Second Division, Department of Pathology.

Address for reprints: Hisayoshi Fujiwara, M.D., Third Division, Department of Internal Medicine, Faculty of Medicine, Kyoto University, 54 Kawaracho, Shogoin, Sakyo-ku, Kyoto 606, Japan.

Received for publication February 2, 1976. 
(A)

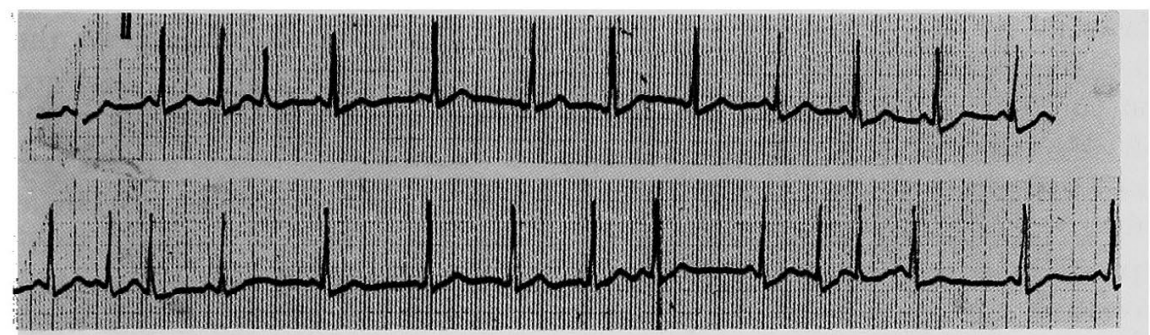

(B)

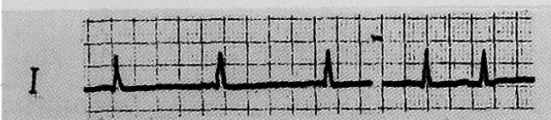

II

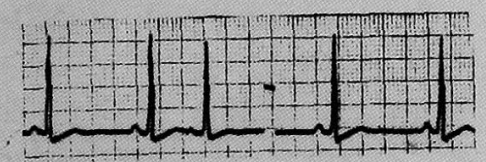

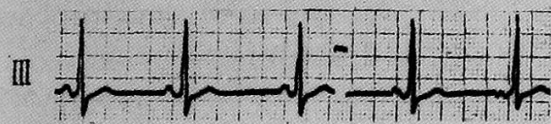

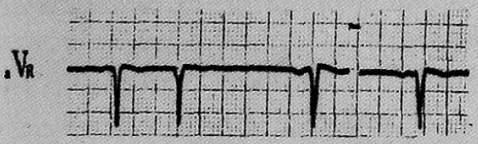

$. V+2$

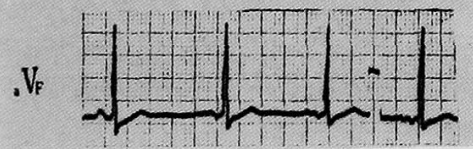

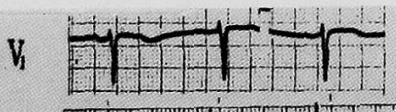

V
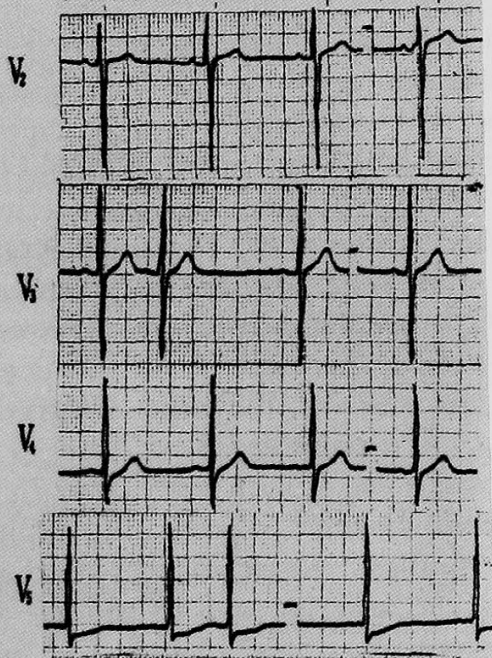

V.

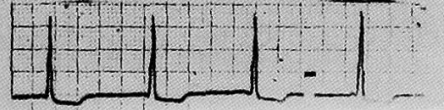

(C)

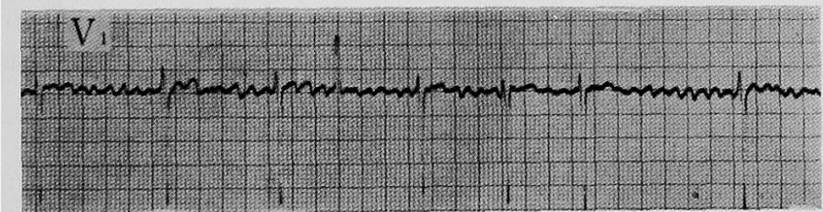

Fig. 1. 57 yr. F. A) ECG on April 24, 1973 : multifocal atrial tachycardia. B) ECG on admission (3/11/1974): APC, changing contour of the $P$ wave, LVH, abnormal $Q$ wave in lead aVL, flat or inverted T, and ST depression. C) ECG of June 2, 1974: atrial fibrillation.

(Fig. 1-A). On November 30, 1973, numbness, pain, and coldness suddenly developed in the left arm; the left brachial arterial pulsation disappeared. EGG showed transient atrial fibrillation with rapid ventricular response. Persistent atrial fibrillation occurred in February, 1974, and lasted till early March of the same year when normal sinus rhythm was spontaneously restored. Two days later the above saddle embolism-like episode occurred. There was no apparent history of hypertension or rheumatic heart disease prior to admission.

Physical examination on admission revealed a nervous small woman who was 
obviously ill. Blood pressure was $132 / 66$ at the right brachial artery, there was no engorgement of the cervical veins, and chest X-ray revealed an enlarged heart contour (CTR: 0.62) and a slight pulmonary congestion was evident. ECG (Fig. 1-B) showed left ventricular hypertrophy in normal sinus rhythm with occasional atrial premature contractions and changing contour of the $\mathrm{P}$ waves. The axis was $+65^{\circ}$. The $T$ waves were flat in leads I, II, III, aVL, aVF, and $V_{5,6}$, and the ST depressions were noted in leads $\mathrm{V}_{5,6}$, II, III, and aVF. In lead aVL, abnormal $Q$ wave had been evident for the past 1 year, indicating old high lateral wall infarction.

Two to 3 weeks later, the ECG demonstrated atrial fibrillation (Fig. 1-G), which persisted until death. A clinical diagnosis of saddle embolism at the bifurcation of the terminal abdominal aorta, old thromboembolism in the left brachial artery and ischemic heart disease with high lateral infarction was made. Atrial infarction was suggested because of atrial arrhythmias combined with ventricular infarction, although deviation of P-Ta segment was not detected,

Urokinase, digitalis, papaverine hydrochloride, and acenocoumarol were prescribed. The above signs and symptoms improved gradually.

On August 31, 1974, the patient collapsed on her way from the lavatory. The ECG immediately recorded showed a ventricular fibrillation. Seven hours later the patient died despite all efforts at resuscitation.

At autopsy, the heart weighed $515 \mathrm{Gm}$ with significant concentric hypertrophy. At a point of $1.5 \mathrm{~cm}$ distal to the origin of the left circumflex artery there was a severe stenosis with more than $75 \%$ narrowing of the lumen secondary to atherosclerotic changes. Sectioning of left ventricular heart muscle revealed mottled whitish fibrotic areas in the lateral and anterior wall.

The anterior wall of the left atrium near the appendage showed an area of discoloration, approximately $1 \times 1 \mathrm{~cm}$ in size. A reddish mural thrombus, approximately $1 \times 1.5 \times 1 \mathrm{~cm}$ (Fig. 2) was found to be loosely adhered by 3 fine pedicles

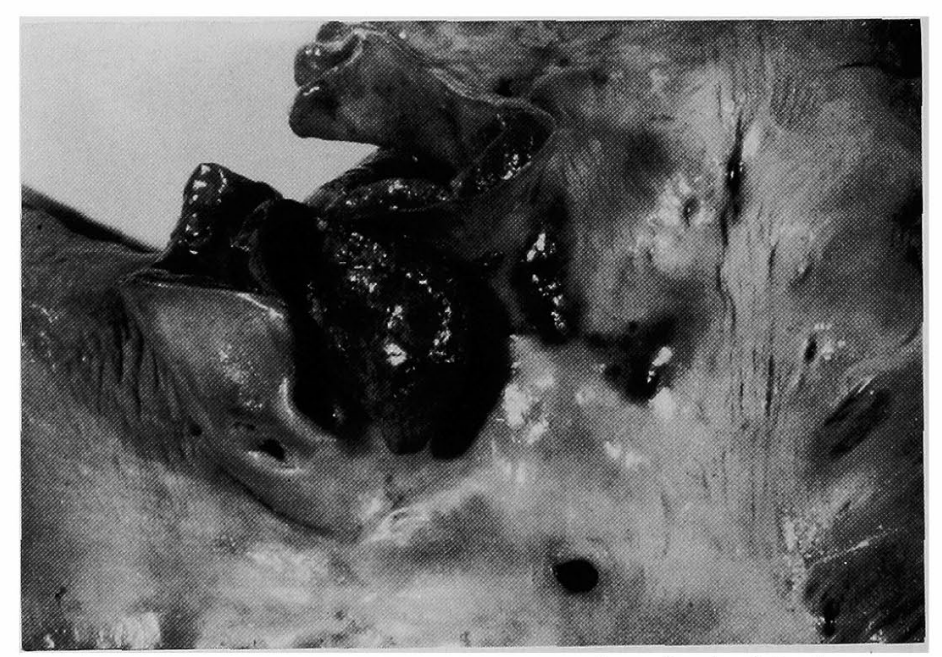

Fig. 2. 57 yr. F. Mural thrombus, approximately $1 \times 1.5 \times 1 \mathrm{~cm}$, near the appendage in the LA. There was atrial infarction directly beneath that thrombus. 


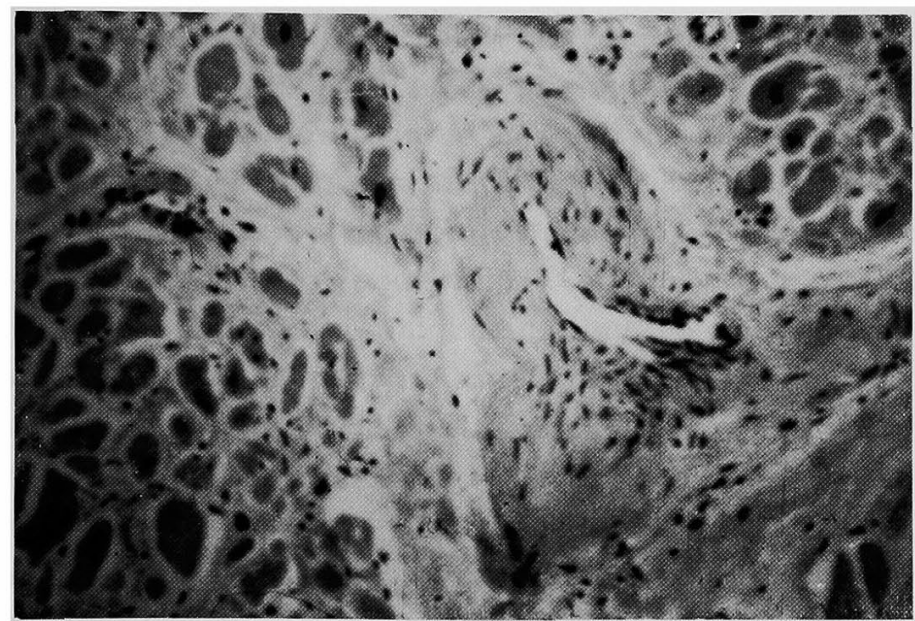

Fig. 3. 57 yr. F. Microscopical section taken from the left atrial wall directly beneath the mural thrombus revealed poorly defined muscle fibers with fragmentation, hypertrophy and degeneration as well as fibrosis and infiltration of the mononuclear cells. A small artery showed marked atherosclerosis.

to the left atrial endocardium beneath the above stated discolorated area. The endocardium of both ventricles and right atrium was smooth and shiny.

Microscopical sections taken from the left atrial wall beneath the thrombus disclosed an old infarction which revealed fragmentation, hypertrophy, and degeneration of muscle fibers, infiltration of mononuclear cells, and fibrosis (Fig. 3). There was no significant change in the sinus node and SA nodal artery.

The mitral valve showed no pathological change. There were thromboembolic occlusions of left brachial artery and of the lower portion of abdominal aorta with multiple infarctions of both kidneys.

\section{Discussion}

Systemic arterial thromboembolism and left ventricular mural thrombi are common complications of ventricular myocardial infarction. Left atrial infarction and left atrial mural thrombi are indeed rare as most atrial infarction as well as atrial thrombi occur in the right atrium. ${ }^{1)}$ According to Wartman and Hellerstein, ${ }^{2)}$ left-sided cardiac thrombi were observed in $34 \%$ of cases of myocardial infarction and all thrombi were located in the left ventricle. But autopsy revealed that a large thrombus over the left atrial infarcted area was loosely adhered to the endocardium through 3 fine pedicles and could be easily detached. It seems likely that a new thrombus formed repeatedly from the residual pedicles, resulting in multiple systemic thromboembolism. Such findings indicate that systemic arterial thromboembolism 
may occur due to atrial infarction which is often overlooked at antemortem as well as postmortem examination. The fact that saddle embolism occurred 2 days after normal sinus rhythm was restored from atrial fibrillation indicates that the change in cardiac rhythm is an important factor related to possible embolism. It has been generally considered that the prognosis of atrial infarction was not dependent on the prognosis of atrial infarction itself, but rather on the prognosis of ventricular infarction accompanying atrial infarction except for atrial rupture, and that the diagnosis was of little clinical importance. $^{1)}$ Five autopsy cases of atrial infarction have been reported by Kimura et $\mathrm{a}^{3}{ }^{3}$ and 3 of these 5 did not demonstrate a ventricular infarction. One of the 3 patients, who had had a right atrial infarction, had also StokesAdams syndrome and died of congestive heart failure following various atrial arrhythmias, one died of right atrial rupture and the cause of death in the last one was not reported. The other 2 patients who had both right atrial and left ventricular infarction died of congestive heart failure and one had a Stokes-Adams syndrome due to atrial standstill. Young and Koeniga) also reported a case of isolated right atrial infarction followed by sudden death, presumably due to atrial arrhythmia.

In our present work we found a severe complication of systemic arterial thromboembolism and such led to the conclusion that the diagnosis of atrial infarction is indeed of clinical importance as severe complications such as Stokes-Adams syndrome and systemic arterial thromboembolism may also be present.

\section{AGKNOWLEDGEMENTS}

Gratitude is due to Dr. H. Ogawa for referral of the patient, to Dr. K. Ishikawa for the clinical care, and to M. Ohara for assistance with the manuscript.

\section{REFERENCES}

1. Friedberg CK: Diseases of the Heart. 3rd ed, Saunders Co, Philadelphia and London, p 860,1966

2. Wartman WB, Hellerstein HK: The incidence of heart disease in 2,000 consecutive autopsies. Ann Int Med 28: 41, 1948

3. Kimura M, Mashiba U, Kitsuki K, Ogino T, Onuki J, Katayama K, Koie O: An autopsy case of atrial infarction. Heart 6: 799, 1973 (in Japanese)

4. Yung EW, Koenig ABS: Auricular infarction. Am Heart J 28: 287, 1944 